
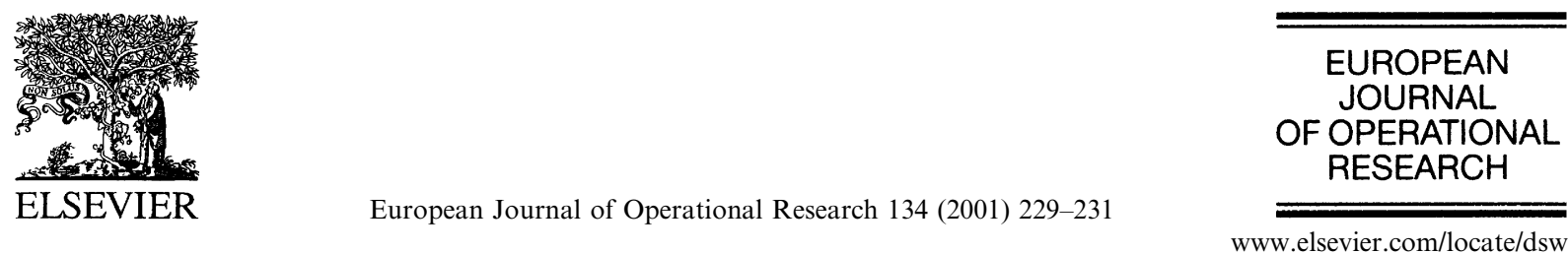

\title{
Editorial
}

\section{Financial modelling in the new millennium}

\author{
Jaap Spronk ${ }^{\mathrm{a}, *}$, Nico van der Wijst ${ }^{\mathrm{b}}$ \\ ${ }^{a}$ Department of Finance, Erasmus Universiteit Rotterdam, P.O. Box 1738, 3000 DR Rotterdam, The Netherlands \\ ${ }^{\mathrm{b}}$ Department of Industrial Economics and Technology Management, Norwegian University of Science and Technology, \\ 7491 Trondheim, Norway
}

\section{Introduction}

For a profession as young as financial modelling, the prospects at the turn of the millennium are exciting. We are in a new and rapidly developing area of research. The vast majority of the theoretical insights that we try to model date from the last few decades. Many of the algorithms and electronic aids we use are of similar or even more recent date. Most of the problems we try to solve with our modelling arose with the advent of the complex and interrelated societies that we live in today. In many ways we have only just begun.

In many other ways financial modelling is, of course, deeply rooted in centuries of science. Interestingly, some authors e.g. Bernstein [1], place the beginning of modern science at the point in time where the future ceased to be the exclusive realm of fortune-tellers and prophets and became the subject of scientific scrutiny. The birth of probability theory is seen by many as this watershed between ancient and modern science and that makes financial modelling a clear exponent of modern science. Allegedly, probability theory began its existence some 350 years ago when a rich French nobleman called upon two prominent mathematicians of the day,

\footnotetext{
${ }^{*}$ Corresponding author. Tel.: +31-10-408-1428; fax: +31-10452-6399.

E-mail address: spronk@few.eur.nl (J. Spronk).
}

Pascal and Fermat, to help him with his gambling. The analogy with the rocket scientists being called upon by today's rich men in Wall Street to help them with their investment problems is too tempting not to be used here. The other obvious association is with gambling and this has haunted users of probability theory from the very beginning. In his well-known book, Cramér [2] refers to the episode with the French nobleman as the "very humble beginning' and feels obliged to make the apologising remark that gambling was a fashionable habit at the time, apparently not too much restricted by law. The analogy with modern day hedge funds, too little restricted according to some, is again tempting. It also points, through the recent collapse involving a well-known hedge fund, at an essential element of financial modelling: that human behaviour (and human error) is somehow part of whatever model we make. Humans are never will-less entities in models, they have ever-changing expectations and an everlasting desire to beat the system (or the model). That too goes back all the way to the beginning: the French nobleman is not likely to have gambled to get the fair expected return.

\section{Contents of this issue}

The long roots of financial modelling do not imply a lack of current challenges and we find 
some fine examples of them in the papers in this special issue. Together, they give a good impression of its richness and variety, ranging from bank branch efficiency to option pricing and asset liability management for a pension fund using stochastic programming.

In the first paper, Hartman et al. analyse the productivity of providing bank services. In a combined analysis of 'doing things right' and 'doing the right things', they decompose inefficiencies of bank branches in their technical and allocative components. Besides making a scientific contribution, this paper also shows how comparatively new techniques as DEA can be brought to bear on practical and highly actual decision making in banking.

Bradfield and Raubenheimer investigate the consequences of restricted leverage on the allocation of risky assets. They show that constraints on leverage will lead to a different composition of the optimal portfolio compared with an unconstraint situation, thus highlighting a hitherto sparsely explored aspect of Markowitz' portfolio theory.

Bounds on option prices are investigated in the Basso and Pianca paper. Rather than making strict assumptions about the underlying security and market to obtain a specific price, they use widely accepted hypotheses regarding the behaviour of agents facing risk to derive an interval for the price of a portfolio of European options. The interval can be used to test for mispricings in the market, which is particularly useful for non-standard options that are not officially quoted.

The last three papers have stochastic programming as a common theme and they show an interesting variation in the aspects and applications that are considered.

First of all, Dupačová and Bertocchi give an analysis of the bond portfolio management problem using a stochastic programming model and data from the Italian bond market. They use simulation studies to analyse the sensitivity of the optimal value of the bond portfolio management problem to selected scenarios of interest rates.

In the second stochastic modelling paper, Kouwenberg studies the scenario generating process in the context of an asset liability management model for a pension fund. Random sampling, ad- justed random sampling and a tree fitting approach are used to construct event trees. The conclusions, based on rolling horizon simulations, indicate that the random sampling approach may lead to excessive trading and spurious profits. Asset mix switching is considerably reduced by the adjusted random sampling procedure, which drastically improved the performance of the ALM model. The adjusted random sampling procedure is, in turn, slightly outperformed by a tree fitting procedure.

The third stochastic programming paper by Høyland and Wallace also applies an asset liability management model, to analyse the legal regulations for life insurance businesses in Norway, with highly critical conclusions as to these regulations.

\section{Plans for the feature issues on financial modelling}

Keeping abreast with developments in a rapidly changing area of research like financial modelling requires frequent contact with the work and ideas of other interested people from both science and practice. For the past 13 years, the EURO Working Group on Financial Modelling has been a successful platform for this contact. The meetings of this Working Group, organised twice per year ${ }^{1}$, have become an important place for presenting papers, exchanging ideas and start joint research projects. EJOR's Feature Issues on Financial Modelling intend to follow up on these meetings with a Feature Issue after every meeting. The purpose of this is to bring more regularity in the Feature Issues and thereby starting a mutually enforcing cycle of publications and presentations. The purpose is by no means to exclude papers from outside the EURO Working Group Meetings. On the contrary, all papers that fit the editorial policy ${ }^{2}$ will remain welcome as before.

\footnotetext{
${ }^{1}$ The previous two meetings were in Vienna and Trondheim (Norway), the next two will be in New York and Vilnius (Lithuania).

${ }^{2}$ Papers on financial modelling are solicited that help to solve financial-economic decision problems in practice. They may relate to new insights, both theoretical and empirical, into the decision maker's environment, new tools and the integration of these tools within frameworks for decision making.
} 
The Nobel prizes recently awarded to option pricing theorists complete a by now impressive list of laureates in finance. With this, finance can be said to have come of age as a scientific discipline. Ahead of us lies the challenge of bringing the discipline to a prosperous mature life. The EURO Working Group Meetings and EJOR's Feature Issues on Financial Modelling intend to play their part in that.

\section{References}

[1] P.L. Bernstein, Against the Gods, the Remarkable Story of Risk, Wiley, New York, 1996.

[2] H. Cramér, The Elements of Probability Theory and Some of its Applications, Wiley, New York, 1955. 